\title{
Lower Esophageal Sphincter-preserving Anastomosis Is an Acceptable Reconstruction Method Following Laparoscopy-assisted Proximal Gastrectomy
}

\author{
DONG JIN KIM ${ }^{1}$ and WOOK KIM ${ }^{2}$ \\ ${ }^{1}$ Department of Surgery, St. Paul's Hospital, College of Medicine, \\ The Catholic University of Korea, Seoul, Republic of Korea; \\ ${ }^{2}$ Department of Surgery; Yeouido St. Mary's Hospital, College of Medicine, \\ The Catholic University of Korea, Seoul, Republic of Korea
}

\begin{abstract}
Background/Aims: Our study aimed to compared 3-year results following laparoscopy-assisted proximal gastrectomy (LAPG)-double tract anastomosis (DTA) and lower esophageal sphincter-preserving $L A P G$ (LES-LAPG). Patients and Methods: Nine patients who underwent LES-LAPG and 23 LAPG-DTA were compared regarding 3-year follow-up complications, the anemiarelated parameter and albumin levels. Results: During the 3-year follow-up period, no patient (0\%) in the LES-LAPG and six patients $(6 / 23,26.1 \%)$ in the LAPG-DTA group developed reflux symptoms. Reflux symptoms were well controlled with PPI in all patients, except for one in the LAPG-DTA group. In the laboratory study, there were no differences in serum ferritin and vitamin B12 levels between the two groups. However, mean hemoglobin and albumin levels were significantly higher in the LES-LAPG group from 18 months postoperatively. Conclusion: LES$L A P G$ provided acceptable results for the prevention of reflux esophagitis. Moreover, LES-LAPG may be advantageous for anemia prevention and nutritional status improvement compared to LAPG-DTA.
\end{abstract}

According to a nationwide survey in Korea, the incidence of early gastric cancer (EGC) and proximal gastric cancer among all gastric cancer increased from $57.6 \%$ and $13.4 \%$ in 2009 to $61.0 \%$ and $16.0 \%$ in 2014 , respectively $(1,2)$. In contrast, proximal gastrectomy was still performed in

Correspondence to: Wook Kim, MD, Ph.D., Department of Surgery, Yeouido St. Mary's Hospital, College of Medicine, The Catholic University of Korea, 10, 63-ro, Yeongdeungpo-gu, Seoul, 07345, Republic of Korea. Tel: +82 237791175, Fax: +82 27869114, email:kimwook@catholic.ac.kr

Key Words: Stomach neoplasm, gastrectomy, anastomosis, esophagitis.
$1.1 \%$ of all surgical procedures in Korea from 2004 to 2014 due to anastomosis-related complications $(1,3)$. To overcome these problems, several types of reconstruction methods are used following laparoscopy-assisted proximal gastrectomy (LAPG) (4-7). One of them, double tract anastomosis (DTA), has become one of the most popular procedures, and its superiority compared to that of total gastrectomy has been reported with respect to nutritional and laboratory parameters (8-10). In addition, DTA can be performed with a single-port procedure as well as intracorporeal anastomosis $(11,12)$. However, strong evidence for the acceptance of DTA as an optimal anastomosis method following LAPG is still lacking. DTA needs three anastomoses, including esophagojejunostomy, which is the most difficult and problematic type, in addition to gastrojejunostomy, and jejunojejunostomy. Moreover, how much ingested food with nutrients will go through normal physiologic food passage is uncertain because of the existence of two potential pathways.

In 2010, we introduced the concept of lower esophageal sphincter preservation when we performed near total gastrectomy with jejunal pouch interposition (13). Thereafter, we adopted this concept for the anastomosis procedure following LAPG, yielding lower esophageal sphincterpreserving LAPG (LES-LAPG), which makes a single anastomosis with a circular stapler (7). LES-LAPG showed promising short-term results in preventing reflux esophagitis. Additionally, it provides a single pathway, which guarantees physiological flow of all ingested nutrients. Although LESLAPG seems to be a better way of anastomosis for nutritional aspects following LAPG theoretically, long-term status should be compared to those of other types of anastomosis regarding reflux esophagitis and nutritional parameters. In the present study, the 3-year results of LESLAPG including follow-up anemia and nutrition-related laboratory data were compared with those of LAPG-DTA. 


\section{Patients and Methods}

Patient and study design. Among 598 patients who underwent curative intent laparoscopic gastrectomy from March 2009 to December 2016, 41 patients who underwent LAPG were included in the study. Because proximal gastrectomy was indicated for patients with clinical stage I, we only included 32 patients with clinical stage I disease, excluding those with clinical stage II who underwent proximal gastrectomy at their request. Nine patients who underwent LES-LAPG were compared with 23 patients who underwent LAPG-DTA regarding clinicopathological characteristics including sex, age, American society of Anesthesiologist (ASA) score, body mass index (BMI), tumor location, and pathological results. Early and long-term surgical outcomes were also compared, including early in-hospital complications, and especially those related to anastomosis such as reflux esophagitis status by symptom, need for proton pump inhibitor (PPI), and stricture that needed endoscopic balloon dilatation.

All patients were followed-up regularly every 6 months. At every visit, hemoglobin, iron-related parameters, and vitamin B12 levels were examined. The 3-year laboratory data were compared between the two groups including hemoglobin, serum ferritin, vitamin B12, and serum albumin levels. In addition, weight change from baseline was compared between the two groups. Analysis of overall survival from the date of surgery was also performed.

This study was approved by the Institutional Review Board with approval number SC18RESI0020.

Surgical procedures for LES-LAPG and LAPG-DTA. The surgical procedure was performed according to previously described methods for each anastomosis technique $(7,10)$.

Indication of anastomosis for LES-LAPG and LAPG-DTA. Basically, the indication for LES-LAPG and LAPG-DTA is similar. However, while LAPG-DTA is indicated for whole proximal EGC located at the proximal third, LES-LAPG has a relatively narrow indication. More than 2.5 to $3 \mathrm{~cm}$ distance from the esophagogastric junction to the proximal border of the tumor is indicated for LES-LAPG.

Statistical analysis. All statistical analyses were performed using PASW statistics version 18.0 (IBM, Armonk, NY, USA). Continuous variables were described as mean with standard deviation and compared using the Student's $t$-test. Nominal variables were compared using Chi-square and Fisher's exact tests. Survival analysis was performed with KaplanMeier model and log-rank test. All statistical analyses were considered significant when the $p$-value was less than 0.05 .

\section{Results}

There was no statistical difference between the two groups with respect to the clinicopathologic characteristics including sex, age, ASA score, and clinical and pathologic staging (Table I).

Although tumor size and resection margin did not differ significantly, the tumor location was significantly different between the two groups: While all tumors in the LES-LAPG group were located in the upper body, $65.2 \%$ of those in the LAPG-DTA group were located in the cardia. Regarding the pathological tumor staging, most patients (78\%) had stage I
Table I. Clinicopathological comparison between patients treated with lower esophageal sphincter-preserving laparoscopy-assisted proximal gastrectomy (LES-LAPG) and those treated with laparoscopy-assisted proximal gastrectomy with double tract anastomosis (LAPG-DTA).

\begin{tabular}{|c|c|c|c|}
\hline Variable & $\begin{array}{l}\text { LES-LAPG } \\
\qquad(\mathrm{n}=9)\end{array}$ & $\begin{array}{l}\text { LAPG-DTA } \\
\quad(\mathrm{n}=23)\end{array}$ & p-Value \\
\hline \multicolumn{4}{|l|}{ Gender } \\
\hline Male & $6(66.7 \%)$ & $19(82.6 \%)$ & \multirow[t]{2}{*}{0.327} \\
\hline Female & $3(33.3 \%)$ & $4(17.4 \%)$ & \\
\hline \multicolumn{4}{|l|}{ Age, years } \\
\hline Mean \pm SD & $60.7 \pm 7.6$ & $68.5 \pm 10.2$ & 0.047 \\
\hline \multicolumn{4}{|l|}{ ASA, n (\%) } \\
\hline 1 & $4(44.4 \%)$ & $10(43.5 \%)$ & \multirow[t]{3}{*}{0.816} \\
\hline 2 & $5(55.6 \%)$ & $5(55.6 \%)$ & \\
\hline 3 & $0(0 \%)$ & $1(4.3 \%)$ & \\
\hline \multicolumn{4}{|l|}{ BMI, $\mathrm{kg} / \mathrm{m}^{2}$} \\
\hline Mean \pm SD & $24.0 \pm 1.7$ & $23.9 \pm 3.3$ & 0.956 \\
\hline \multicolumn{4}{|c|}{ Tumor location, n (\%) } \\
\hline Cardia & $0(0 \%)$ & $15(65.2 \%)$ & \multirow[t]{3}{*}{0.001} \\
\hline Upper body & $9(100 \%)$ & $6(26.1 \%)$ & \\
\hline Mid-body & $0(0 \%)$ & $2(8.7 \%)$ & \\
\hline \multicolumn{4}{|l|}{ cTstage, n (\%) } \\
\hline $\mathrm{T} 1$ & $8(88.9 \%)$ & $20(87.0 \%)$ & \multirow[t]{2}{*}{0.882} \\
\hline $\mathrm{T} 2$ & $1(11.1 \%)$ & $3(13.0 \%)$ & \\
\hline \multicolumn{4}{|l|}{ Tumor size, $\mathrm{cm}$} \\
\hline Mean \pm SD & $2.2 \pm 1.4$ & $2.2 \pm 1.4$ & 0.935 \\
\hline \multicolumn{4}{|l|}{ pTstage, n (\%) } \\
\hline $\mathrm{T} 1$ & $7(77.8 \%)$ & $19(82.6 \%)$ & \multirow[t]{3}{*}{0.417} \\
\hline $\mathrm{T} 2$ & $2(22.2 \%)$ & $2(8.7 \%)$ & \\
\hline $\mathrm{T} 3$ & $0(0 \%)$ & $2(8.7 \%)$ & \\
\hline \multicolumn{4}{|l|}{ pNstage, n (\%) } \\
\hline NO & $9(100 \%)$ & $21(91.3 \%)$ & \multirow[t]{4}{*}{0.659} \\
\hline N1 & $0(0 \%)$ & $1(4.3 \%)$ & \\
\hline $\mathrm{N} 2$ & $0(0 \%)$ & $0(0 \%)$ & \\
\hline N3 & $0(0 \%)$ & $1(4.3 \%)$ & \\
\hline \multicolumn{4}{|l|}{ pStage, n (\%) } \\
\hline Ia & $7(77.8 \%)$ & $18(78.3 \%)$ & \multirow[t]{4}{*}{0.548} \\
\hline $\mathrm{Ib}$ & $2(22.2 \%)$ & $2(8.7 \%)$ & \\
\hline IIa & $0(0 \%)$ & $1(4.3 \%)$ & \\
\hline $\mathrm{IIb}$ & $0(0 \%)$ & $2(8.7 \%)$ & \\
\hline \multicolumn{4}{|c|}{ Proximal margin, $\mathrm{cm}$} \\
\hline Mean \pm SD & $2.5 \pm 1.3$ & $2.1 \pm 1.5$ & 0.402 \\
\hline \multicolumn{4}{|c|}{ Distal margin, $\mathrm{cm}$} \\
\hline Mean \pm SD & $5.4 \pm 1.5$ & $4.5 \pm 2.3$ & 0.326 \\
\hline \multicolumn{4}{|c|}{ Retrieved LNs, n } \\
\hline Mean \pm SD & $26.6 \pm 13.5$ & $32.8 \pm 15.8$ & 0.308 \\
\hline
\end{tabular}

ASA: American Society of Anesthesiology grading; BMI: body mass index; LN: lymph nodes.

disease and staging did not differ significantly between the two groups.

In the short-term surgical result, there was one anastomotic leakage in each group at the gastrogastrostomy and esophagojejunostomy sites. One patient in the LAPG-DTA group developed bleeding, which was managed with conservative care. One patient in the LES-LAPG group developed anastomotic stricture requiring balloon dilatation (Table II). 


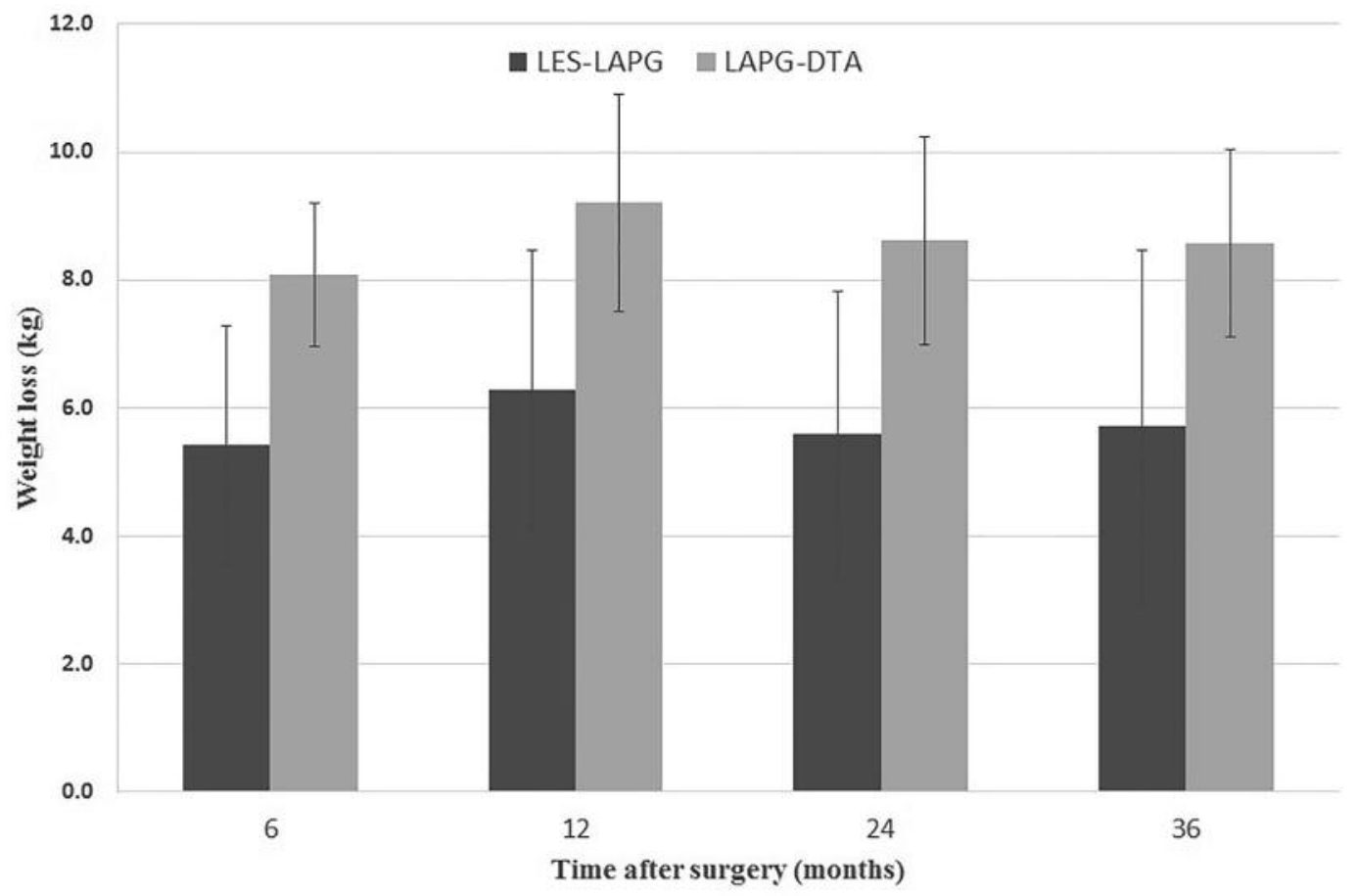

Figure 1. Amount of weight loss from preoperative weight in patients treated with lower esophageal sphincter-preserving laparoscopy-assisted proximal gastrectomy (LES-LAPG) and those treated with laparoscopy-assisted proximal gastrectomy with double tract anastomosis (LAPG-DTA). Data are the mean $\pm S D$.

During the 3-year follow-up period, no patient $(0 \%)$ and six patients $(6 / 23,26.1 \%)$ in the LES-LAPG and LAPGDTA groups, respectively, developed reflux symptoms and required PPI. Reflux symptoms were well controlled with PPI in all patients except one in the LAPG-DTA group.

In the 3-year follow-up, regarding nutritional results, the amount of weight change and various laboratory data were compared. While there was no significant difference in weight change between the two groups, the degree of weight loss was less in the LES-LAPG group compared with that in the LAPG-DTA group (Figure 1). In the laboratory study, there were no differences in serum iron, ferritin, and vitamin B12 levels between the two groups. However, mean hemoglobin and albumin levels were significantly higher in the LES-LAPG group from 18 months to 3 years postoperatively (Figure 2).

There was no recurrence in the LES-LAPG group, and one patient died due to a cardiac event at the 1-year postoperative period. The other patients lived for more than 5 years. In the LAPG-DTA group, one patient developed peritoneal metastasis and died due to disease progression. In the survival analysis, there was no difference in the 5-year overall survival between the two groups (LES-LAPG vs. LAPG-DTA, 89.9\% vs. 90\%, $p=0.857$ ) (Figure 3).
Table II. Early and 3-year surgical outcomes in patients treated with lower esophageal sphincter-preserving laparoscopy-assisted proximal gastrectomy (LES-LAPG) and those treated with laparoscopy-assisted proximal gastrectomy with double tract anastomosis (LAPG-DTA).

\begin{tabular}{lllr}
\hline Variable & $\begin{array}{l}\text { LES-LAPG, } \\
\mathrm{n}(\%)(\mathrm{n}=9)\end{array}$ & $\begin{array}{l}\text { LAPG-DTA, } \\
\mathrm{n}(\%)(\mathrm{n}=23)\end{array}$ & $p$-Value \\
\hline $\begin{array}{lll}\text { Early complication } \\
\quad \text { Overall }\end{array}$ & $1(11.1 \%)$ & $7(30.4 \%)$ & 0.256 \\
$\quad$ Pulmonary & $0(0 \%)$ & $4(17.4 \%)$ & \\
Leakage & $1(11.1 \%)$ & $1(4.3 \%)$ & \\
Bleeding & $0(0 \%)$ & $1(4.3 \%)$ & \\
$\quad$ Other & $0(0 \%)$ & $1(4.3 \%)$ & \\
Long-term & & & \\
$\quad$ Reflux symptoms & $0(0 \%)$ & $6(26.1 \%)$ & 0.150 \\
$\quad$ PPI medication & $0(0 \%)$ & $6(26.1 \%)$ & 0.150 \\
$\quad$ Stricture & $1(11.1 \%)$ & $0(0 \%)$ & 0.281 \\
Recurrence & $0(0 \%)$ & $1(4.3 \%)$ & $>0.999$ \\
Death & $1(11.1 \%)$ & $2(8.7 \%)$ & $>0.999$ \\
\hline
\end{tabular}

Pulmonary: grade I $(n=2)$, grade IIIa $(n=1)$, grade Iva $(n=1)$; Leakage: all grade IIIa; Bleeding: grade II; Other: hiccup (grade I).

\section{Discussion}

This study analyzed the long-term follow-up results following different types of anastomosis after proximal gastrectomy. It is 

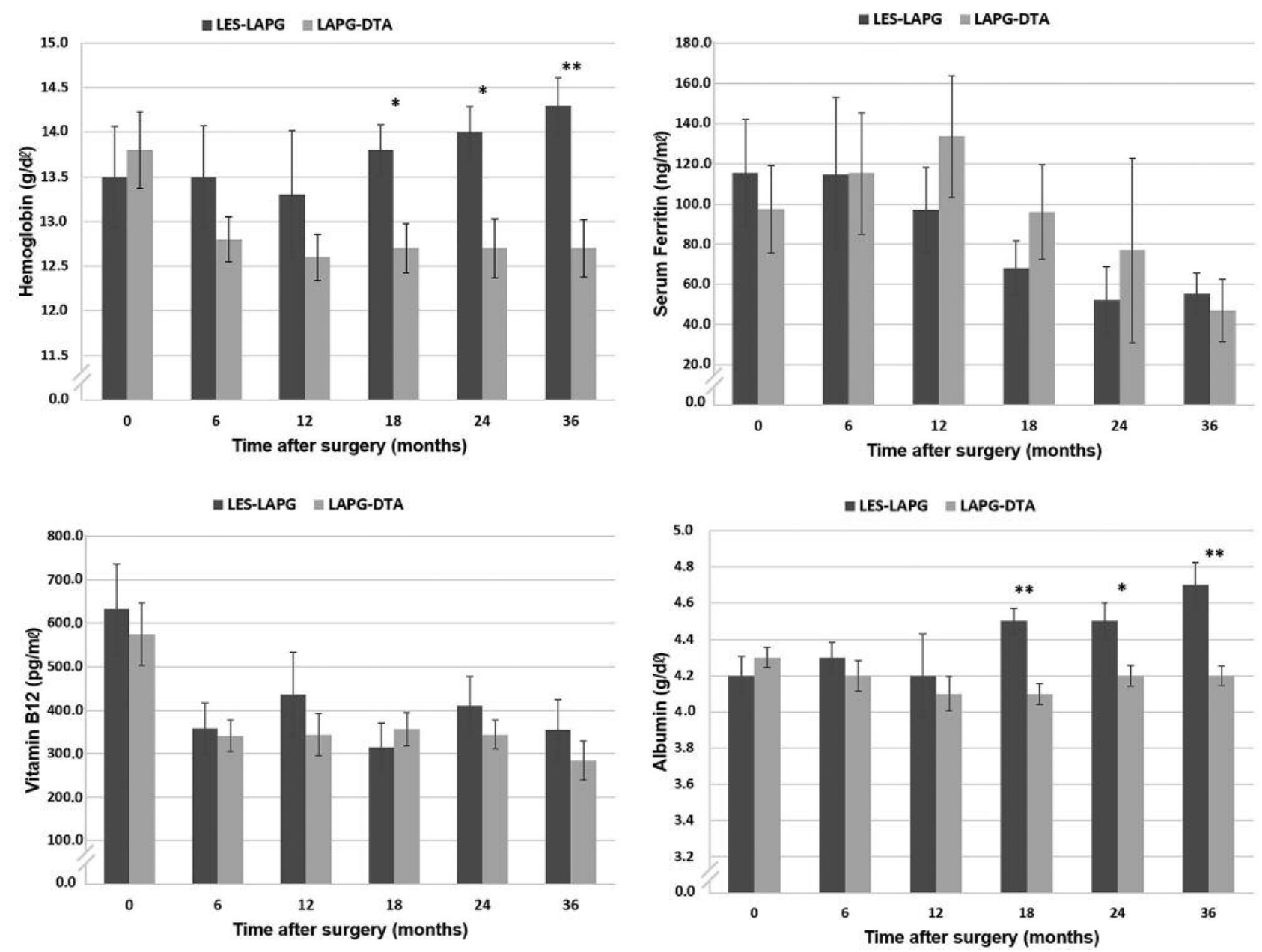

Figure 2. Laboratory comparisons between nine patients treated with lower esophageal sphincter-preserving laparoscopy-assisted proximal gastrectomy (LES-LAPG) and 17 treated with laparoscopy-assisted proximal gastrectomy with double tract anastomosis (LAPG-DTA) until postoperative year 3. Significantly different at $* p<0.05$ and $* * p<0.01$. Data are the mean $\pm S D$.

obvious that many surgical procedures are performed to substitute for total gastrectomy for patients with proximal EGCs. Even though there are many studies showing its advantages, proximal gastrectomy was only performed in $1.0 \%$ of all gastric cancer-related surgeries in Korea in 2014 (1).

Previously, the result of proximal gastrectomy was dismal due to the high incidence of reflux esophagitis and anastomotic stricture. An et al. reported anastomotic stenosis and reflux rates of $38.2 \%$ and $29.2 \%$, respectively, in their patients who underwent proximal gastrectomy with esophagogastric tube reconstruction (14). In other Korean studies, these rates were reported as $46.5 \%$ and $48 \%$, respectively. Moreover, reflux esophagitis was reported in $100 \%$ of patients after proximal gastrectomy $(15,16)$.

However, recent studies dealing with novel anastomosis techniques reported relatively favorable results regarding reflux esophagitis. Ahn et al. reported a $4.6 \%$ incidence of anastomotic stricture and reflux symptom in 43 patients who underwent LAPG-DTA with a mean follow-up of 21.6 months (17). In a Japanese study, the incidence of reflux esophagitis after LAPG with double flap reconstruction was $2.3 \%$ in 43 patients (18). However, this procedure needs a long operative time and several intracorporeal suturing procedures.

The study reported by our team also showed the incidence of reflux esophagitis as $0 \%$ and $11.8 \%$ in the LES-LAPG and LAPG-DTA groups, respectively, in the short-term followup result $(7,10)$.

Among the novel anastomosis techniques, DTA was frequently compared with total gastrectomy $(9,10)$. In these reports, proximal gastrectomy was not inferior in reflux esophagitis and superiority in nutritional parameters. However, DTA has definite disadvantages. Despite the feasibility of the 
intracorporeal anastomosis technique, it needs three anastomoses including esophagojejunostomy, which is one of the most difficult and problematic types of anastomosis (19).

There are no studies of long-term follow-up results with laboratory changes comparing DTA with other types of anastomosis. Moreover, using a gastric emptying study using radioisotope, Ahn et al. revealed that two-thirds of ingested food passes through the physiological pathway. However, this is not guaranteed. In contrast, LES-LAPG is a method wherein $100 \%$ of ingested food passes through the duodenum, and this difference might explain the difference in hemoglobin and albumin levels between the two procedures.

One of the most important results in this study is that there was no significant gastroesophageal reflux in patients who underwent LES-LAPG even without sufficient distance from the distal esophagus and remnant stomach. Basically, the lower esophageal sphincter is not a true sphincter, such as the pylorus and anal sphincter. Instead, it consists of four elements: intrinsic esophageal circular muscles, gastric sling fiber, diaphragmatic sling fiber, and length of the intraabdominal esophagus. Considering the reconstruction method, LES-LAPG can preserve three of these components except the gastric sling fiber. On the contrary, LAPG-DTA can save nothing especially when a linear stapler is used in performing esophagojejunostomy. Because of traction of the thoracic esophagus, esophagojejunostomy anastomosis can usually be fully or partially located above the diaphragmatic sling fiber. This might be the main difference in the preservation of LES and reflux esophagitis following proximal gastrectomy.

The other point is the nutritional aspect. The laboratory follow-up results showed superiority of LES-LAPG regarding hemoglobin and albumin levels from 18 months postoperatively. To reduce any bias, we included patients who had laboratory data available for more than 1 year, and who were free from chemotherapy. For this reason, only 17 patients in the LAPG-DTA group and all patients in the LESLAPG group were included. For iron-related metabolism, the serum ferritin level reflects the amount of stored iron. In the comparison of ferritin level, there was no difference between the two groups. Although we cannot explain the exact mechanism of increased hemoglobin level in LES-LAPG regardless of the difference in ferritin and vitamin B12 levels, we can assume that there are likely to be other nutritional factors affecting the difference in hemoglobin and albumin levels after 18 months. In addition, the degree of weight loss was lower in the LES-LAPG than the LAPGDTA group (Figure 2). The similar results were found in a Japanese study comparing between 35 patients who underwent total gastrectomy and 49 who underwent proximal gastrectomy (20).

There are some limitations to this study. Firstly, this study was retrospectively designed, and a relatively small

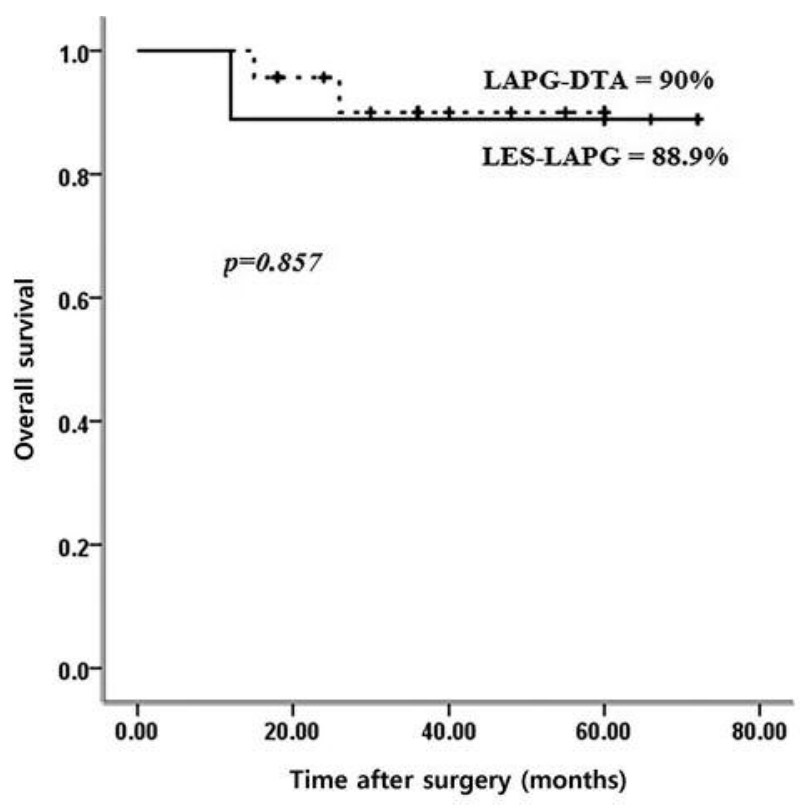

Figure 3. Overall survival analysis comparing patients treated with lower esophageal sphincter-preserving laparoscopy-assisted proximal gastrectomy (LES-LAPG) and those treated with laparoscopy-assisted proximal gastrectomy with double tract anastomosis (LAPG-DTA).

number of patients was included. While we were performing LES-LAPG, intracorporeal anastomosis was introduced. At that time, we were unable to avoid making an epigastric midline wound when we performed LESLAPG. For this reason, we considered the DTA procedure, and consequently there were only nine patients who underwent LES-LAPG. However, we developed intracorporeal LES-LAPG and plan to collect more data. Secondly, there was a discrepancy in the indication for application of both procedures. The indication for LAPGDTA includes that for LES-LAPG. We compared the two procedures since we wanted to elucidate the exact role of the anastomosis shape itself. There might be more consideration for the terminology of our procedure. A small portion of the cardia should be preserved in order to save the LES; thus, proximal gastrectomy might not be adopted when preserving the cardia. However, it can be considered as a reverse shape to that observed in pylorus-preserving gastrectomy. In conclusion, LES-LAPG showed acceptable results regrading prevention of reflux esophagitis. Moreover, LES-LAPG might have advantages in prevention of anemia and improvement of nutritional status.

\section{Conflict of Interest}

All Authors have nothing to disclose. 


\section{Ethical Standards}

All procedures followed were in accordance with the ethical standards of the responsible Institutional Review Committee on human experimentation and with the Helsinki Declaration of 1964 and later versions. Informed consent or substitute for it was obtained from all patients included in the study.

\section{References}

1 The Information Committee of Korean Gastric Cancer Association: Korean Gastric Cancer Association Nationwide Survey on Gastric Cancer in 2014. J Gastric Cancer 16: 131-140, 2016.

2 Jeong O and Park YK: Clinicopathological features and surgical treatment of gastric cancer in South Korea: The results of 2009 nationwide survey on surgically treated gastric cancer patients. J Gastric Cancer 11: 69-77, 2011.

3 The Information Committee of the Korean Gastric Cancer Association: 2004 Nationwide Gastric Cancer Report in Korea. J Korean Gastric Cancer Assoc 7: 8, 2007.

4 Ahn SH, Lee JH, Park DJ and Kim HH: Laparoscopy-assisted proximal gastrectomy for early gastric cancer is an ugly duckling with unsolved concerns: oncological safety, late complications, and functional benefit. Gastric Cancer 16: 448-450, 2013.

5 Aihara R, Mochiki E, Ohno T, Yanai M, Toyomasu Y, Ogata K, Ando H, Asao T and Kuwano H: Laparoscopy-assisted proximal gastrectomy with gastric tube reconstruction for early gastric cancer. Surg Endosc 24: 2343-2348, 2010.

6 Hoshikawa T, Denno R, Ura H, Yamaguchi K and Hirata K: Proximal gastrectomy and jejunal pouch interposition: evaluation of postoperative symptoms and gastrointestinal hormone secretion. Oncol Rep 8: 1293-1299, 2001.

7 Kim DJ, Lee JH and Kim W: Lower esophageal sphincterpreserving laparoscopy-assisted proximal gastrectomy in patients with early gastric cancer: a method for the prevention of reflux esophagitis. Gastric Cancer 16: 440-444, 2013.

8 Jung DH, Ahn SH, Park DJ and Kim HH: Proximal gastrectomy for gastric cancer. J Gastric Cancer 15: 77-86, 2015.

9 Jung DH, Lee Y, Kim DW, Park YS, Ahn SH, Park DJ and Kim HH: Laparoscopic proximal gastrectomy with double tract reconstruction is superior to laparoscopic total gastrectomy for proximal early gastric cancer. Surg Endosc 31: 3961-3969, 2017.

$10 \mathrm{Kim}$ DJ and Kim W: Laparoscopy-assisted proximal gastrectomy with double tract anastomosis is beneficial for vitamin B12 and iron absorption. Anticancer Res 36: 4753-4758, 2016.
11 Lee CM, Park DW, Jung DH, Jang YJ, Kim JH, Park S and Park SH: Single-Port Laparoscopic proximal gastrectomy with double tract reconstruction for early gastric cancer: Report of a case. $\mathbf{J}$ Gastric Cancer 16: 200-206, 2016.

12 Yang K, Bang HJ, Almadani ME, Dy-Abalajon DM, Kim YN, Roh KH, Lim SH, Son T, Kim HI, Noh SH and Hyung WJ: Laparoscopic proximal gastrectomy with double-tract reconstruction by intracorporeal anastomosis with linear staplers. J Am Coll Surg 222: e39-45, 2016.

13 Kim DJ, Hur H, Jeon HM and Kim W: Near-total gastrectomy preserving the lower esophageal sphincter followed by jejunal pouch interposition as a treatment for upper gastric cancer. J Korean Surg Soc 78: 290-297, 2010.

14 An JY, Youn HG, Choi MG, Noh JH, Sohn TS and Kim S: The difficult choice between total and proximal gastrectomy in proximal early gastric cancer. Am J Surg 196: 587-591, 2008.

15 Katsoulis IE, Robotis JF, Kouraklis G and Yannopoulos PA: What is the difference between proximal and total gastrectomy regarding postoperative bile reflux into the oesophagus? Dig Surg 23: 325-330, 2006.

16 Kim JH, Park SS, Kim J, Boo YJ, Kim SJ, Mok YJ and Kim CS: Surgical outcomes for gastric cancer in the upper third of the stomach. World J Surg 30: 1870-1876; discussion 1877-1878, 2006.

17 Ahn SH, Jung DH, Son SY, Lee CM, Park DJ and Kim HH: Laparoscopic double-tract proximal gastrectomy for proximal early gastric cancer. Gastric Cancer 17: 562-570, 2013.

18 Hayami M, Hiki N, Nunobe S, Mine S, Ohashi M, Kumagai K, Ida S, Watanabe M, Sano T and Yamaguchi T: Clinical outcomes and evaluation of laparoscopic proximal gastrectomy with double-flap technique for early gastric cancer in the upper third of the stomach. Ann Surg Oncol 24: 1635-1642, 2017.

$19 \mathrm{Kim} \mathrm{DJ}$, Lee JH and Kim W: Comparison of the major postoperative complications between laparoscopic distal and total gastrectomies for gastric cancer using Clavien-Dindo classification. Surg Endosc 29: 3196-3204, 2015.

20 Ichikawa D, Komatsu S, Kubota T, Okamoto K, Shiozaki A, Fujiwara $\mathrm{H}$ and Otsuji E: Long-term outcomes of patients who underwent limited proximal gastrectomy. Gastric Cancer 17: 141-145, 2013
Received November 5, 2018

Revised November 17, 2018

Accepted November 21, 2018 